\title{
Neutrophil to Lymphocyte Ratio and Platelet to Lymphocyte Ratio as Marker of Disease Activity in Rheumatoid Arthritis
}

\author{
REHAB M. HELAL, M.Sc.*; MOHAMMED H. EL-NAGGAR, M.D.*; MOHAMED K. ZAHRA, M.D.** and \\ NASHWA M. ABO EL-NASR, M.D.* \\ The Departments of Internal Medicine* and Clinical Pathology**, Faculty of Medicine, Tanta University
}

\begin{abstract}
Background: RA is a chronic disease that carries a substantial burden for both the individual and society. The individual burden results from musculoskeletal deficits, with attendant decline in physical function, quality of life, and cumulative comorbid risk. The socioeconomic burden, aside from major direct medical costs, is a consequence of functional disability, reduced work capacity, and decreased societal participation. Efforts to establish the diagnosis early, initiate treatment promptly, and design novel treatment strategies to control inflammation and reduce or prevent consequent damage are paramount.
\end{abstract}

Aim of Study: Is to evaluate the Neutrophil-Lymphocytic Ratio (NLR) and Platelet-Lymphocyte Ratio (PLR) as markers of disease activity in rheumatoid arthritis.

Methods: This study included 50 patients with rheumatoid arthritis divided into three groups; group 1 including 30 patients with clinically active disease, group 2 including 20 patients without clinical activity and group 3 including 20 apparently healthy age and gender matched volunteers as controls. CBC, ESR, CRP, Anti-CCP, RF were done to all participants.

Results: The NLR and PLR of RA patients was significantly higher compared to that of the controls. Furthermore, a significant correlation between NLR and the DAS 28 score was observed.

Conclusion: NLR and PLR is a promising markers in the assessment of RA disease activity.

Key Words: Neutrophil Lymphocyte Ratio (NLR) - Platelet Lymphocyte Ratio (PLR) - Rheumatoid arthritis.

\section{Introduction}

RHEUMATOID Arthritis (RA) a chronic and systemic autoimmune diseases with inflammation of synovial joints; it results in the progressive damage of joint, especially when inflammation is persistent [1]

Correspondence to: Dr. Rehab M. Helal, The Department of Internal Medicine, Faculty of Medicine, Tanta University
Approximately, $75 \%$ of untreated patients resulted in disability within three years. During the development and progression of the diseases, hematological changes are often occurred prior to morphological changes to reflect the clinical status. Hematological change is a kind of feedback to the systemic inflammation. Complete Blood Count (CBC) is used to detect the variation of different components in peripheral blood for diagnosing diseases of the hematopoietic system [2]

Disease-activity assessment is crucial for RA management, since it could greatly affect the clinical decision. Current disease-activity assessment mainly depends on clinical symptoms or signs, laboratory tests and questionnaires. Laboratory tests are very much preferred due to the advantage of less observer variations. C-Reactive Protein (CRP) and Erythrocyte Sedimentation Rate (ESR) are two well-recognized inflammatory indices and have been widely used for RA disease-activity assessment [3].

Although all neutrophils, mast cells, and B and T lymphocytes play established roles in the pathogenesis of RA, it is believed that the Neutrophil/ Lymphocyte Ratio (NLR) is a particularly important measure of systemic inflammation. NLR can be readily computed. Patients with RA may present with increasing platelet counts during active stages of the disease [4].

Parameters of hemogram, especially including immune system elements, have a significant place in the assessment of various diseases and/or hints of diseases. Among the immune system elements, neutrophils, lymphocytes and platelets play a role in the control of inflammation, while also undergoing changes secondary to inflammation [5] . 


\section{Subjects and Methods}

The study was performed on fifty patients with RA fulfilling American College of Rheumatology (ACR) 1987 classification criteria and European League Against Rheumatism (EULAR) 2010 criteria. They were admitted at Internal Medicine Department at Tanta University Hospitals or attending at its outpatient clinic of Rheumatology and Clinical Immunology Unit in the period from April 2017 to March 2018 and 20 healthy age-and gender-matched volunteers as control group. All participant provided informed written consent and the study was approved by Tanta Faculty of Medicine Ethical Committee.

The participants were divided into the following groups:

- Group (1): Thirty rheumatoid arthritis patients with clinically active disease.

- Group (2): Twenty rheumatoid arthritis patients without clinical activity.

- Group (3): Twenty apparently healthy age and gender matched healthy controls.

\section{Inclusion criteria:}

The diagnosis of RA will be established according to the American college of Rheumatology (ACR) 1987 classification criteria and European League Against Rheumatism (EULAR) 2010 criteria for early diagnosis of RA.

\section{Exclusion criteria:}

Patients with cardiac disease, cerebrovascular disease, hematologic disease (such as chronic myelogenous leukemia, thrombocytosis), known chronic liver and kidney diseases, infectious diseases, malignant tumor diseases, hypertension, diabetes, metabolic syndrome and other autoimmune diseases (such as ankylosing spondylitis and systemic lupus erythematosus).

All participants in this study were subjected to: Thorough history taking, full clinical examination, laboratory investigations in the form of: Compelete blood count, Erythrocyte Sedimentation Rate (ESR), C-Reactive Protein (CRP), Anti-Cyclic Citrullinated Peptide (Anti-CCP), Rheumatoid Factor (RF).

\section{Sampling and laboratory investigations:}

Sampling and all laboratory investigations were done in Clinical Pathology Department, Tanta University Hospitals.

Compelete blood count was performed using ERMA INC (model PCE-210N) full automatic blood cell counter, with examination of Giemsastained peripheral blood smears for differential leucocyte count.

Erythrocyte Sedimentation Rate (ESR) by Westergren method.

C Reactive Protein (CRP) was determined using immunoassay.

Rheumatoid Factor (RF) was assessed using RF Latex which was a slide agglutination test for the qualitative and semiqantitative detection of RF in human serum. latex particles coated with human gamma globulin were agglutinated when mixed with samples which contain RF.

Anti-cyclic citrullinated peptide (Anti-CCP).

Statistical analysis of the data:

Data were fed to the computer and analyzed using IBM SPSS software package Version 20.0. For quantitative data, the Shapiro-Wilk test for normality was performed. For normally distributed data, values were expressed as mean \pm standard deviation and one-way ANOVA was performed for comparison between the studied and control groups. For data that were not normally distributed median and interquartile range (expressed as 25 th- 75 th percentiles) were calculated and Mann-Whitney and Kruskall-Wallis tests were used. In case of one-way ANOVA and Kruskall-Wallis tests, pairwise comparison between the three groups was done using the suitable post hoc tests. As regards qualitative data, Pearson's Chi square test was used to examine association between two variables. Additionally, Spearman's rank correlation was done to test associations of the studied variables with NLR and PLR. ROC-curve was used for assessment of sensitivity and specificity. Significance of the obtained results was judged at the $p$-value $<0.05$.

\section{Results}

Comparison between the studied groups showed statistical significance as regard to hemoglobin, platelet count, neutrophil count, WBC count, ESR1, CRP, RF, DAS-28 and Anti-CCP. In contrary comparison showed no statistical significance as regard age, sex and lymphocytes. As shown in (Table 1).

As for NLR, it was significantly higher in group 1 in comparison to the other two groups.

As for PLR, it was significantly higher in group 1 in comparison to group 3 . As shown in (Table 2). 
In this study NLR showed positive correlation with statistical significance with neutrophil, ESR1, RF, Anti CCP, DAS 28, PLR and morning stiffness ( $p$-value $<0.001), \mathrm{CRP}$ ( $p$-value 0.001$)$, WBCs ( $p$ value 0.003 ). And showed negative correlation with statistical significance with lymphocyte ( $p$ value $<0.001)$. There was no significant correlation between studied patients and following parameters: Age, HB level and platelet. As shown in (Table 3).

In this study PLR showed positive correlation with statistical significance with platelet ( $p$-value $0.049)$. And negative correlation with statistical significance with lymphocyte $(p$-value $<0.001)$ and morning stiffness ( $p$-value 0.015 ). There was no significant correlation between studied patients and following parameter: Age, HB level, neutrophil, WBCs, ESR1, CRP, RF, Anti CCP and DAS 28. as shown in (Table 4).

The ROC curve showed that (considering a cutoff value of $\geq 3.02 \%$ for NLR and $\geq 112.59 \%$ for PLR): The sensitivity of NLR as a marker of RA activity was $90 \%$, while it's specificity was $85 \%$ with $86 \%$ accuracy. The sensitivity of PLR as a marker of RA activity was $70 \%$, while it's specificity was $50 \%$ with $60 \%$ accuracy. As shown in (Table 5) and Figs. (1,2).

Table (1): Comparison between the studied groups.

\begin{tabular}{lllll}
\hline Parameter & $\begin{array}{l}\text { Group 1 } \\
(\mathrm{n}=30)\end{array}$ & $\begin{array}{l}\text { Group 2 } \\
(\mathrm{n}=20)\end{array}$ & $\begin{array}{l}\text { Group 3 } \\
(\mathrm{n}=20)\end{array}$ & $p$ \\
\hline Age in years & $36.27 \pm 5.43$ & $34.7 \pm 6.54$ & $36.1 \pm 5.55$ & 0.619 \\
Sex (female/male) & $22 / 8$ & $14 / 6$ & $15 / 5$ & 0.882 \\
Hb gm/dl & $10.33 \pm 0.93$ & $10.57 \pm 1.12$ & $12.04 \pm 1.11$ & $<0.001$ \\
Platelet X10 3 cell/mm3 & 260000 & 221500 & 163500 & $<0.001$ \\
Neutrophil & $7191.6 \pm 2434.61$ & $4639 \pm 1767.48$ & $3689.05 \pm 1483.17$ & $<0.001$ \\
Lymphocyte & 1920 & 2209 & 2166 & 0.089 \\
WBCs X10 3 cell/mm3 & $9660 \pm 3167.03$ & $7070 \pm 2203.41$ & $7117.50 \pm 1969.99$ & $<0.001$ \\
ESR1 & 38 & 5 & 4 & $<0.001$ \\
CRP & 13.1 & 6 & 4 & $<0.001$ \\
RF & 83 & 40 & 3 & $<0.001$ \\
Anti-CCP & 156 & 59.5 & 5 & $<0.001$ \\
PLR & 148.48 & 114.81 & 85.4 & 0.002 \\
NLR & 3.62 & 1.97 & 1.73 & $<0.001$ \\
DAS28 & 4.79 & 2.33 & N.A & $<0.001$ \\
\hline
\end{tabular}

Table (2): Comparison between studied groups as regards PLR and NLR.

\begin{tabular}{|c|c|c|c|c|c|c|}
\hline & \multicolumn{4}{|c|}{ Groups } & \multicolumn{2}{|c|}{ Kruskal-Wallis test } \\
\hline & $\begin{array}{c}\text { Group } 1 \\
\mathrm{~N}=30\end{array}$ & $\begin{array}{c}\text { Group } 2 \\
\mathrm{~N}=20\end{array}$ & $\begin{array}{c}\text { Group } 3 \\
\mathrm{~N}=20\end{array}$ & $\begin{array}{l}\text { Total } \\
\mathrm{N}=70\end{array}$ & $x^{2}$ & $p$-value \\
\hline \multicolumn{7}{|l|}{ PLR: } \\
\hline Minimum & 58.33 & 56.56 & 43.58 & 43.58 & 12.225 & $0.002 *$ \\
\hline Maximum & 505.05 & 176.18 & 193.69 & 505.05 & & \\
\hline Median & 148.48 & 114.81 & 85.4 & 121.05 & & \\
\hline IQR & $101.61-205.82$ & 88.96- 134.66 & $64.09-138.64$ & $87.27-156.1$ & & \\
\hline Mean rank & 44.83 & 32 & 25 & & & \\
\hline
\end{tabular}

Pairwise comparison revealed: Group 1 versus group $2(p=0.087)$, group 1 versus group $3(p=0.002 *)$, group 2 versus group $3(p=0.830)$.

\begin{tabular}{lllllll}
\hline$N L R:$ & & & & & \\
Minimum & 2.06 & 0.75 & 1.29 & 0.75 & 42.687 & $<0.001 *$ \\
Maximum & 9 & 3.95 & 2.7 & 9 & & \\
Median & 3.62 & 1.97 & 1.73 & 2.5 & \\
IQR & $3.21-4.24$ & $1.40-2.68$ & $1.48-1.98$ & $1.76-3.5$ & \\
Mean rank & 53.63 & 25.1 & 18.7 & & \\
\hline
\end{tabular}

Pairwise comparison revealed: Group 1 versus group $2(p<0.001 *)$, group 1 versus group $3(p<0.001 *)$, group 2 versus group $3(p=0.960)$. IQR: Interquartile Range. 
Table (3): Correlations of NLR with other variables of the studied groups.

\begin{tabular}{|c|c|c|c|c|c|c|}
\hline & \multicolumn{6}{|c|}{ NLR } \\
\hline & \multicolumn{2}{|c|}{$\begin{array}{c}\text { Group } 1 \\
\mathrm{~N}=30\end{array}$} & \multicolumn{2}{|c|}{$\begin{array}{c}\text { Group } 2 \\
\mathrm{~N}=20\end{array}$} & \multicolumn{2}{|c|}{$\begin{array}{r}\text { Total } \\
\mathrm{N}=50\end{array}$} \\
\hline & rs & $p$-value & $r$ & $p$-value & rs & $p$-value \\
\hline Age & -0.066 & 0.73 & 0.083 & 0.727 & 0.106 & 0.464 \\
\hline $\mathrm{Hb}$ & 0.197 & 0.297 & -0.226 & 0.337 & -0.085 & 0.558 \\
\hline Platelets & 0.143 & 0.45 & -0.23 & 0.329 & 0.174 & 0.228 \\
\hline WBCs & -0.012 & 0.949 & 0.535 & $0.015 *$ & 0.407 & $0.003 *$ \\
\hline Neutrophils & 0.228 & 0.225 & 0.694 & $0.001 *$ & 0.590 & $<0.001 *$ \\
\hline Lymphocytes & -0.651 & $<0.001 *$ & -0.483 & $0.031 *$ & -0.58 & $<0.001 *$ \\
\hline PLR & 0.457 & $0.011 *$ & 0.267 & 0.254 & 0.527 & $<0.001 *$ \\
\hline ESR1 & -0.182 & 0.335 & 0.004 & 0.986 & 0.554 & $<0.001 *$ \\
\hline CRP & 0.065 & 0.735 & -0.192 & 0.417 & 0.451 & $0.001 *$ \\
\hline $\mathrm{RF}$ & -0.029 & 0.878 & 0.449 & $0.047 *$ & 0.525 & $<0.001 *$ \\
\hline Anti CCP & -0.156 & 0.409 & -0.062 & 0.795 & 0.546 & $<0.001 *$ \\
\hline DAS28 & -0.094 & 0.623 & 0.084 & 0.724 & 0.598 & $<0.001 *$ \\
\hline Morning stiffness (hours) & 0.226 & 0.228 & -0.373 & 0.105 & 0.532 & $<0.001 *$ \\
\hline
\end{tabular}

Table (4): Correlations of PLR with other variables of the studied groups.

\begin{tabular}{lccccccc}
\hline & \multicolumn{7}{c}{ PLR } \\
\cline { 2 - 8 } & \multicolumn{2}{c}{$\begin{array}{c}\text { Group 1 } \\
\text { N=30 }\end{array}$} & \multicolumn{2}{c}{$\begin{array}{c}\text { Group 2 } \\
\text { N=20 }\end{array}$} & \multicolumn{2}{c}{$\begin{array}{c}\text { Total } \\
\text { N=50 }\end{array}$} \\
\cline { 2 - 9 } & \multicolumn{2}{c}{ rs } & $p$-value & rs & $p$-value & rs & $p$-value \\
\hline Age & 0.257 & 0.17 & 0.017 & 0.994 & 0.239 & 0.094 \\
Hb & -0.154 & 0.416 & 0.189 & 0.426 & -0.067 & 0.645 \\
Platelets & 0.426 & $0.019^{*}$ & 0.326 & 0.16 & 0.418 & $0.003^{*}$ \\
WBCs & -0.553 & $0.002^{*}$ & 0.177 & 0.455 & -0.145 & 0.315 \\
Neutrophils & -0.419 & $0.021^{*}$ & 0.16 & 0.51 & -0.025 & 0.886 \\
Lymphocytes & -0.693 & $<0.001 *$ & -0.123 & 0.606 & -0.605 & $<0.001 *$ \\
ESR1 & -0.229 & 0.225 & -0.481 & $0.032^{*}$ & 0.168 & 0.224 \\
CRP & -0.259 & 0.185 & 0.245 & 0.297 & 0.179 & 0.214 \\
Rheumatoid factor & -0.104 & 0.585 & -0.075 & 0.755 & 0.143 & 0.32 \\
Anti CCP & -0.21 & 0.248 & 0.078 & 0.744 & 0.256 & 0.073 \\
DAS28 & -0.329 & 0.076 & -0.359 & 0.12 & 0.162 & 0.262 \\
Morning stiffness (hours) & 0.249 & 0.185 & -0.106 & 0.567 & -0.180 & $0.015^{*}$ \\
\hline
\end{tabular}

Table (5): The best cut off, sensitivity and specificity for prediction of the disease activity by NLR and PLR.

\begin{tabular}{lcccccc}
\hline & Cut off & Sensitivity & Specificity & Accuracy & AUC & $p$-value \\
\hline NLR & $>3.02$ & $90 \%$ & $85 \%$ & $86 \%$ & 0.92 & $>0.001 *$ \\
PLR & $\geq 112.59$ & $70 \%$ & $50 \%$ & $60 \%$ & 0.7 & $0.017 *$ \\
\hline
\end{tabular}

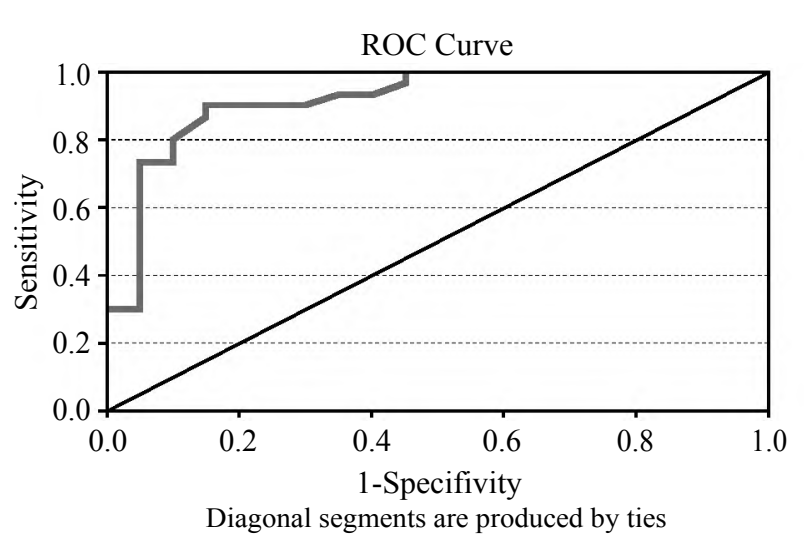

Fig. (1): ROC curve for prediction of the disease activity by NLR.

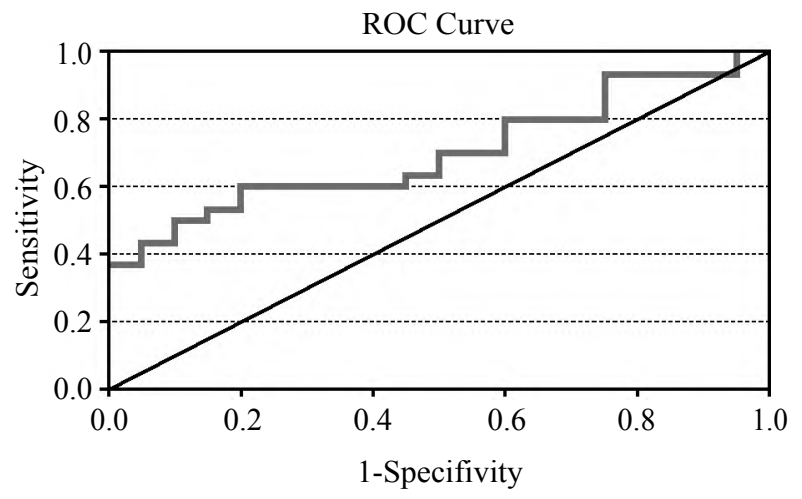

Fig. (2): ROC curve for prediction of the disease activity by PLR. 


\section{Discussion}

Rheumatoid Arthritis (RA) is an autoimmune and chronic inflammatory disorder. Peripheral immune-mediated reactions are pivotal in the pathogenesis of RA, and both the innate and adaptive immune systems are involved in [6].

Rheumatoid Arthritis (RA) is characterized by symmetric joint involvement, erosion and deformity in the joints as a result of synovial inflammation [7].

The etiology of RA remains unknown, multiple mechanisms being involved in the physiopathogenic chain. The heterogeneity of clinical manifestations and variability of therapeutic response demonstrates the complexity of this disease [8]

Systemic inflammation is associated with alterations in circulating blood cells quantity and composition. Indeed, normochromic anemia, thrombocytosis, neutrophilia, and lymphopenia usually accompany many inflammatory conditions. Therefore, the features of circulating blood cell components can be used for the assessment of inflammatory activity [9].

Wright et al., provided a review about neutrophils contributed to disease development of RA. Such as neutrophils release damaging molecules at inflammatory sites, generate and release immunoregulatory cytokines and chemokines which contribute to progress of inflammation. Exposure of these molecules on their cell surface resulted in apoptosis [10].

Lymphocytes are one of the most important cellular immune. Response, playing an important role in the pathogenesis, progression and prognosis of RA through releasing inflammatory agents [11]

Neutrophils, more than $50 \%$ of which are produced by bone marrow, are at the front line of the defense system. They are responsible for the production of many lytic enzymes, free oxygen radicals, and cytokines [12]

In the "treat to target" recommendations, 3 composite scores for the monitorization of the disease evolution are used: Disease activity score (DAS 28), Simple Disease Activity Index (SDAI) and Clinical Disease Activity Index (CDAI). The disadvantage of these scores is the degree of subjectivity of some of the criteria. Moreover, a significant proportion of the patients with negative inflammatory tests, still have active disease [8].
Early diagnosis and therapeutic interventions or treatment can prevent severe manifestations of the disease in patients suffering from RA during the first months of the disease [12].

In the present study NLR and PLR and their correlation to disease activity in 50 Egyptian patients with RA (30 patients with active disease, 20 patients with in active disease with different scores of clinical activity assessed by the DAS 28 score) were investigated and compared to NLR and PLR in 20 healthy volunteers.

In our study, females represented $73.3 \%$ and males represented $26.7 \%$ of the active group with mean age ( $36.27 \pm 5.43$ years), while in the inactive group, females represented $70 \%$ in comparison with males who represented $30 \%$ with mean age (34.70 \pm 6.54 years). Our study has shown that there was no significant difference between RA patients with active disease and RA patients with inactive disease as regard age ( $p$-value $=0.619)$, gender $(p$ value $=0.882)$. This is in agreement with Mercan et al., (2016) [9] and with Zhang et al., (2016) [2] Also female predominance in our study is in agreement with Mercan et al., (2016) [9] and with Zhang et al., (2016) [2]

In our study, 18 out of 50 RA patients ( $36 \%$ ) had neurological manifestations, 49 (98\%) had hematological manifestations, 34 (68\%) had arthritis, $35(70 \%)$ had arthralgia, $8(16 \%)$ had mucocutaneous manifestations and $4(8 \%)$ had RA deformity.

Our study also has shown that hemoglobin levels were significantly decreased in patients with active disease with mean hemoglobin level (10.33 \pm $0.93 \mathrm{gm} / \mathrm{dl}$ ) and patients with inactive disease with mean hemoglobin level $(10.57 \pm 1.12 \mathrm{gm} / \mathrm{dl})(p$ value $<0.001$ ) for each when compared to healthy controls. This is in agreement with Mercan et al., (2016) [9] who assessed the association between neutrophil/lymphocyte ratio and disease activity in rheumatoid arthritis and ankylosing spondylitis among 136 patients with RA and 117 healthy controls.

In our study, in agreement with Peng et al., (2015) [1] and Fu H et al., (2015) [3], we have found that platelet counts were significantly increased in patients with active disease and in patients with inactive disease when compared to healthy control ( $p$-value $<0.001$ and $p=0.012$ ) respectively. On the other hand, Zhang et al., (2016) [2] and Zengin $\mathrm{O}$ et al., (2018) [13] did not find statistically significant association between patients groups and control group regarding platelet count 
which may be due to study design as their study was a retrospective design.

And also there was significantly increase in white blood cell count in patients with active disease when compared to patients with in active disease and control group ( $p$-value 0.003 and $p=$ 0.004 respectively). This is in agreement with Uslu et al., (2015) [5] and Mercan et al., (2016) [9].

Neutrophil count showed higher values in patients with active disease, patients with inactive disease showed lower values than active group, while neutrophil count in control group was the lowest with statistical significance ( $p$-value $<0.001$ ), these results were in agreement with Peng et al., (2015) [1] and Zengin O et al., (2018) [13] who assessed New inflammatory markers in early rheumatoid arthritis, but the results were in contrast to Zhang et al., (2016) [2] and Mercan et al., (2016) [9] who showed no difference in neutrophil count between the groups.

In the current study, we did not find any significant difference between patients with active disease, patients with inactive disease and control group regarding lymphocyte count. This is in agreement with Uslu et al., (2015) [5] and Mercan et al., (2016) [9]. These results were against to Zhang et al., (2016) [2] and Zengin O et al., (2018) [13] who found a significant difference.

Our study has shown that PLR were significantly increased in patients with active disease with median (148.48) when compared to patients with in active disease with median (114.81) and control group with median (85.4) ( $p$-value $=0.002)$. That is in contrast with Mercan et al., (2016) [9] who found that there was no significant difference between studied groups ( $p$-value $=0.16)$. This is in agreement with Zengin O et al., (2018) [13].

As regard NLR our study has shown that there was significant increase in patients with active disease with median (3.62) when compared to patients with in active disease with median (1.97) and control group with median (1.73) ( $p$-value $<0.001)$. This is in agreement with Zengin $\mathrm{O}$ et al., (2018) [13] and Zhang et al., (2016) [2]

The current study has shown that CRP, 1 st hour ESR, RF and Anti-CCP antibody titre were significantly higher in patients with active disease when compared to patients with inactive disease ( $p$-value $<0.001)$. This is in agreement with Zhang et al., (2016) [2] and Zengin O et al., (2018) [13].
In our study, DAS 28 showed positive correlation with statistical significance with following parameters: RF, Anti CCP, ESR1, CRP and NLR $(p$-value $<0.001)$. This is in agreement with Mercan et al., (2016) [9].

In our study, NLR showed positive correlation with statistical significance with neutrophil, ESR1, RF, Anti CCP, DAS 28 ( $p$-value <0.001), CRP ( $p$ value 0.001) WBCs and showed negative correlation with statistical significance with lymphocyte ( $p$-value $<0.001)$. In agreement with $\mathrm{Fu} \mathrm{H}$ et al., (2015) [3].

There was no significant correlation between studied patients and following parameter: Age, HB level, platelet and Morning stiffness.

In our study, PLR showed positive correlation with statistical significance with lymphocyte $(p$ value $<0.001$ ) and platelet ( $p$-value 0.049). In agreement with $\mathrm{Fu} \mathrm{H}$ et al., (2015) [3].

There was no significant correlation between studied patients and following parameter: Age, HB level, neutrophil, WBCs, ESR1, CRP, RF, DAS 28 and Morning stiffness.

\section{Limitations of the study:}

1- The limited number of patients.

2- We studied only a single time measurement of WBCs count.

3- The variation of NLR and PLR, as a crosssectional study, was not observed dynamically after undergoing effective treatment for patients with RA.

4- The association between NLR and PLR and the severity of RA should be discussed to clarify whether PLR is an indicator estimating the severity for patients with RA.

\section{Conclusion:}

- Neutrophil lymphocyte ratio and platelet lymphocyte ratio are potentially useful noninvasive markers to reflect disease activity in RA patients.

- The possible association between NLR, PLR and outcome of inflammatory arthritis remain to be investigated in prospective long-term studies.

\section{Recommendations:}

- More studies are needed to confirm the role of NLR and PLR in diagnosis of RA.

- More studies focusing on NLR and PLR can be carried out for better evaluation of the role of 
NLR and PLR as markers of disease activity in rheumatoid arthritis.

- Further studies should be done for detecting more markers that can help in assessment of RA activity.

- Future researches for assessing the role of NLR and PLR in other autoimmune disease should be put in mind.

- Large scale further prospective studies are clearly necessary to assess the value of NLR and PLR as prognostic markers to detect disease progression in RA patients and they possible use in monitoring clinical response to therapy.

\section{References}

1- PENG Y.F., CAO L., ZENG Y.H., et al.: Platelet to lymphocyte ratio and neutrophil to lymphocyte ratio in patients with rheumatoid arthritis. Open Medicine, 10 (1), 2015.

2- ZHANG Y., YIN Y., KUAI S., et al.: Combination of neutrophil to lymphocyte ratio and platelet to lymphocyte ratio as diagnostic biomarker for rheumatoid arthritis. International Journal of Clinical and Experimental Medicine, 9 (11): p. 22076-81, 2016.

3- FU H., QIN B., HU Z., et al.: Neutrophil-and platelet-tolymphocyte ratios are correlated with disease activity in rheumatoid arthritis. Clin. Lab., 61 (3-4): p. 269-73, 2015.

4- TEKEOGLU I., GÜROL G., HARMAN H., et al.: Overlooked hematological markers of disease activity in rheumatoid arthritis. International Journal of Rheumatic Diseases, 19 (11): p. 1078-82, 2016.

5- USLU A.U., KÜÇÜK A., SAHIN A., et al.: Two new inflammatory markers associated with Disease Activity
Score-28 in patients with rheumatoid arthritis: Neutrophillymphocyte ratio and platelet- lymphocyte ratio. International Journal of Rheumatic Diseases, 18 (7): p. 731-5, 2015.

6- ABDULKADER R., DHARMAPALAIAH C., ROSE G., et al.: Late-onset neutropenia in patients with rheumatoid arthritis after treatment with rituximab. The Journal of Rheumatology, 41 (5): p. 858-61, 2014.

7- KARIMIFAR M., SALESI M. and FARAJZADEGAN Z.: The association of anti-CCP1 antibodies with disease activity score 28 (DAS-28) in rheumatoid arthritis. Advanced Biomedical Research, 1, 2012.

8- GAVRILĂ B., CIOFU C. and STOICA V.: Biomarkers in rheumatoid arthritis, what is new? Journal of Medicine and Life, 9 (2): p. 144, 2016.

9- MERCAN R., BITIK B., TUFAN A., et al.: The association between neutrophil/lymphocyte ratio and disease activity in rheumatoid arthritis and ankylosing spondylitis. Journal of Clinical Laboratory Analysis, 30 (5): p. 597-601, 2016.

10- VEALE D.J. and FEARON U.: Inhibition of angiogenic pathways in rheumatoid arthritis: Potential for therapeutic targeting. Best Practice \& Research Clinical Rheumatology, 20 (5): p. 941-7, 2006.

11- SCARSI M., ZANOTTI C., CHIARINI M., et al.: Reduction of peripheral blood $\mathrm{T}$ cells producing IFN- $\mathrm{y}$ and IL17 after therapy with abatacept for rheumatoid arthritis. Clinical and Experimental Rheumatology, 32 (2): p. 20410, 2014.

12-WANG J. and ARASE H.: Regulation of immune responses by neutrophils. Annals of the New York Academy of Sciences, 1319 (1): p. 66-81, 2014.

13-ZENGIN O., ONDER M., KALEM A., et al.: New inflammatory markers in early rheumatoid arthritis. Zeitschrift für Rheumatologie, 77 (2): p. 144-50, 2018.

\section{نسبة العدلات إلى الخخلايا الليمفاوية والصفائح الدموية إلى الخلايا الليمفاوية

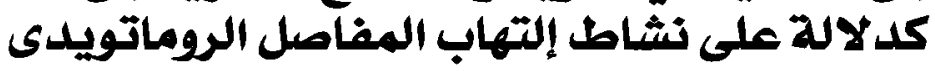

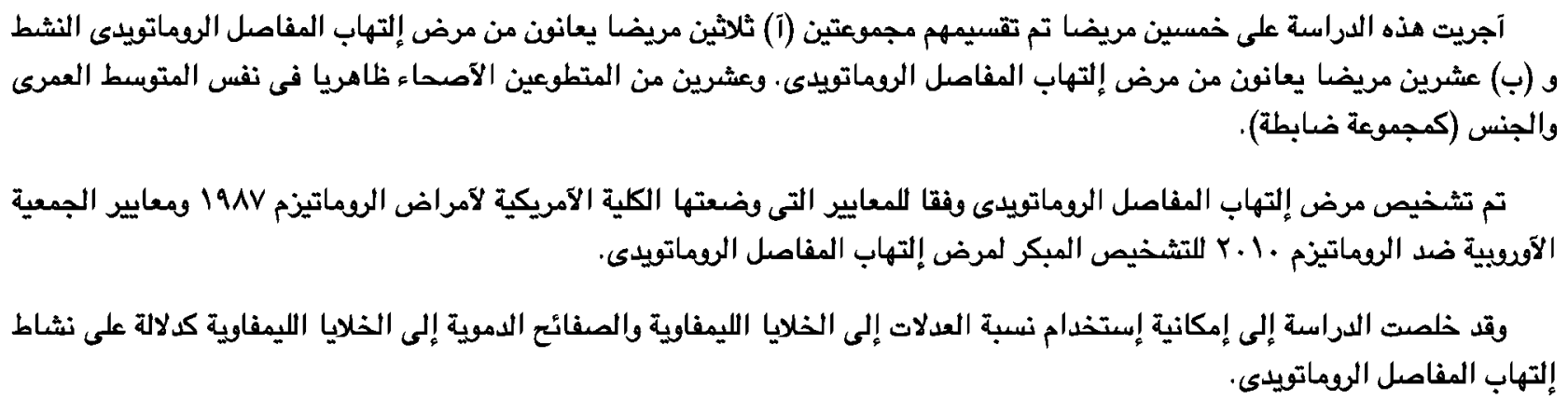

